\title{
Engineering Deans' Support For LGBTQ Inclusion
}

\section{Dr. Erin A. Cech, Rice University}

Erin Cech is an Assistant Professor in the Department of Sociology at Rice University. Before coming to Rice in 2012, Cech was a postdoctoral fellow at Stanford's Clayman Institute for Gender Research. She earned her Ph.D. in Sociology from the University of California, San Diego and B.S. degrees in Electrical Engineering and Sociology from Montana State University. Cech's research seeks to uncover cultural mechanisms of inequality reproduction-particularly gender, sexual identity and racial/ethnic inequality within science and engineering professions. Her current research projects focus on the recruitment and retention of women, racial/ethnic minority and LGBTQ individuals and the role of professional cultures in inequality in STEM.

\section{Prof. Tom J. Waidzunas, Temple University}

Assistant Professor, Department of Sociology, Temple University

\section{Dr. Stephanie Farrell, Rowan University}

Dr. Stephanie Farrell is Professor of Chemical Engineering at Rowan University (USA) and was 2014-15 Fulbright Scholar in Engineering Education at Dublin Institute of Technology (Ireland). She obtained her $\mathrm{PhD}$ in Chemical Engineering from New Jersey Institute of Technology in 1996. Prior to joining the faculty at Rowan in 1998, she was an Assistant Professor of Chemical Engineering and Adjunct Professor of Biomedical Engineering at Louisiana Tech University until 1998. Dr. Farrell has contributed to engineering education through her work in experiential learning, focusing on areas of pharmaceutical, biomedical and food engineering. She has been honored by the American Society of Engineering Education with several teaching awards such as the 2004 National Outstanding Teaching Medal and the 2005 Quinn Award for experiential learning. Stephanie has conducted workshops on a variety of topics including effective teaching, inductive teaching strategies and the use of experiments and demonstrations to enhance learning. 


\section{Engineering Deans' Support For LGBTQ Inclusion}

Over the last several years, scholars have started to investigate processes of disadvantage and exclusion faced by lesbian, gay, bisexual, transgender and queer (LGBTQ) faculty, students and professionals in engineering. Although this research is only beginning to document the contours of these issues, it suggests that successful social change in engineering requires that equality and inclusion be advanced through multiple fronts in engineering education, including the policies and perspectives of engineering administrators and leaders. In this paper, we draw on survey data (currently being collected) from over 40 Deans and Program Directors of U.S. Engineering and Engineering Technology colleges and programs to understand their views on LGBTQ-inclusive practices and policies. In particular, our paper describes (1) deans' assessment of the climate in their college for LGBTQ students and faculty, (2) deans' personal support for LGBTQ equality and inclusion measures (e.g. faculty-wide Safe Zone training, hiring initiatives, inclusion of LGBTQ status in non-discrimination statements in job advertisements) and (3) deans' perception of support among their faculty and students for LGBTQ inclusion measures. These findings provide important insights into the cultural and policy landscapes in engineering education for LGBTQ individuals and the (un)supportiveness of this key group of stakeholders of the promotion of LGBTQ equality. ${ }^{1}$

\section{Introduction}

Considerations of diversity and inclusion have gained increased traction in science, technology, engineering and math (STEM) education broadly and in engineering education specifically over the last several decades [1-3]. ${ }^{2}$ Compared to the amount of scholarship and initiatives aimed at advancing the representation and inclusion of women and racial/ethnic minority students and faculty in engineering, issues of inclusion along the lines of sexual orientation and gender identity/expression have received much less attention [4-5].

Recent research has indicated that lesbian, gay, bisexual, transgender, and queer (LGBTQ) individuals face interactional and institutional processes of disadvantage in STEM, ranging from a lack of LGBTQ-inclusive benefits [6,7], to biases that undermine LGBTQ individuals' treatment and work satisfaction [8], to processes of heterosexism (attitudes and acts of discrimination and prejudice against LGBTQ persons) and heteronormativity (commonly-held assumptions about the appropriateness and naturalness of heterosexual relationships and a strict male/female binary) that undercut LGBTQ persons' integration into the STEM community $[4,9,10]$ and even question their competence as engineers $[4,12]$. This heterosexism and heteronormativity are linked both to broader societal status biases that devalue LGBTQ persons as untrustworthy, immoral, or incompetent $[8,15]$ and to ideologies specific to the culture of engineering, such as "depoliticization" (the strict bracketing of political or cultural issues that are seen as "political" from engineering work) and the "technical/social dualism" (the elevation of "technical" skills and activities over social skills and activities) that devalue and delegitimate

\footnotetext{
${ }^{1}$ This research was supported by a grant from the National Science Foundation (grant 1107074; PI: Stephanie Farrell; Co-PIs: Rocio Chavela Guerra, Erin Cech, Tom Waidzunas, and Adrienne Minerick). Any opinions, findings, and conclusions or recommendations expressed in this material are those of the authors and do not necessarily reflect the views of the National Science Foundation.

${ }^{2}$ The Women in Engineering Division of ASEE was established in 1978 and the Minorities in Engineering Division was established in 1999.
} 
considerations of issues of LGBTQ equality within the context of "real" engineering work [4, $16]$.

Effective action toward undermining these processes of inequality is likely requires from both "bottom-up" or grassroots efforts by groups of students and faculty seeking to facilitate positive change and from "top-down" programs or efforts that are initiated at the institution or school level. Although grassroots action may be particularly influential in bringing about long-term social and cultural changes that make engineering education more inclusive for LGBTQ individuals, within the institutional structure of higher education programs, movements toward inclusion often requires endorsement of (or at least tacit support from) institutional leaders and gatekeepers [10].

In order to understand the supportiveness of key gatekeepers in engineering education toward LGBTQ inclusion efforts, we conducted the first-ever diversity and inclusion survey of deans of engineering and engineering technology colleges and programs $(\mathrm{N}=47)$. We address the following questions in this paper: (1) do deans tend to recognize that LGBTQ inclusion is a problem in their engineering/engineering technology colleges and programs? (2) Do deans personally support LGBTQ inclusion initiatives? (3) Do they believe that the faculty and students in their engineering college or program would support such initiatives? WE describe these three questions in more detail below and then present our methods and results.

(1) Do deans report LGBTQ inclusion problems in their colleges and programs? Our first goal is to understand the extent to which engineering deans report problems with LGBTQ inclusion in their colleges and programs. As research on broader U.S. populations has demonstrated, the motivation to make positive change first requires that individuals recognize a problem in need of being resolved $[13,14]$. Specifically, we are interested in whether deans see instances of exclusion, heteronormativity and heterosexism toward LGBTQ faculty and students in their colleges and programs.

(2) Do deans personally support LGBTQ inclusion efforts? Beyond simply recognizing LGBTQ inclusion issues within their programs or colleges, we are interested in whether deans personally support efforts for LGBTQ inclusion. Specifically, we examine deans' support for a range of initiatives that span different levels of institutional investment, from offering Safe Zone trainings to instigating a job search for openly-LGBTQ engineering faculty. The level of support deans express for such initiatives would indicate where most deans fall on a spectrum from serving as roadblocks for institutional change or to being untapped allies in support of such changes.

(3) Do deans believe that faculty and students would support LGBTQ inclusion efforts? As leaders of their programs and colleges, engineering deans may be unlikely to advance inclusion efforts - even if they personally champion them - if they do not believe such efforts would be backed by engineering faculty and students. As such, we examine deans' perception of the support from their constituents for those LGBTQ inclusion efforts.

The next sections detail our unique survey data and the results from our analysis and then discusses the implications of these findings for scholarship on inequality in STEM and for promotion of LGBTQ inclusion in engineering education and engineering practice. 


\section{Methodology}

We utilize unique survey data of Deans and Program Directors of engineering and engineering technology colleges of programs at U.S. institutions. With permission from the American Society for Engineering Education, the authors sent survey invitations to all 370 Deans and Program Directors affiliated with ASEE's Engineering Deans' Council and Engineering Technology Council. Respondents received the initial invitations via email and received a follow-up reminder by email and by hard-copy, hand-addressed letter. Thus far, 84 people participated in our survey. This yielded a response rate of 23 percent of U.S. deans of engineering colleges and programs, which is on par with online survey response rates and fairly high given that this is a particularly busy population with many demands on their time. Of the total survey participants, 47 of those had data on to all of the measures we use in this analysis and thus are included in our analysis here.

Respondents were asked a series of questions related to their perspective on the climate in their college for LGBTQ faculty and students, their personal support for LGBTQ inclusion initiatives, and their perspective on how supportive engineering students and faculty would be of those same initiatives. In the results section that follows, we present means on our measures of interest. Our small sample size restricts us from using complicated multivariate analyses.

\section{Results}

Table 1 presents descriptive statistics on the deans in our sample. The majority (65\%) of respondents are heads of engineering colleges and programs at public four-year institutions. Consistent with deans of U.S. engineering colleges and programs more broadly, women and persons of color are under-represented among our sample. Approximately five percent of the sample identifies as gay or lesbian; this is slightly higher than existing statistics on sexual minorities in STEM [8], likely due to the survey's focus on diversity and inclusion issues.

\begin{tabular}{|c|c|c|}
\hline & Mean & $S D$ \\
\hline Dean of Public, Four-Year College & .652 & .466 \\
\hline Dean of Private, Non Religiously-Affiliated College & .283 & .429 \\
\hline Dean of Private, Religiously-Affiliated College & .065 & .262 \\
\hline Gender identity: woman & .326 & .474 \\
\hline Gender identity: man & .671 & .474 \\
\hline Gay, lesbian, bisexual or queer & $\sim .050$ & .206 \\
\hline Nonwhite & .136 & .347 \\
\hline
\end{tabular}

Note: The proportion of sexual minority respondents is presented as an approximation to protect confidentiality; the survey sample used here did not include any individuals who identified as transgender.

How do engineering deans characterize the climate of their colleges or programs? A series of questions asked respondents to assess the climate for LGBTQ persons. Specifically, deans were asked whether they agree (1 to 5 likert scale, strongly disagree to strongly agree) with a range of 
statements regarding their assessments of the climate in their college or program. In Table 2, we report the proportion of deans who somewhat or strongly agree with each statement.

Nearly a quarter of deans agree that their colleagues make comments that seem to indicate a lack of awareness of LGBTQ issues, and a fifth report that there seems to be pressure in their engineering college for LGBTQ persons to stay closeted. Fifteen percent feel that LGBTQ persons are pressured to conceal their LGBTQ identity from colleagues, and 11 percent say that LGBTQ employees in their college are expected not to act "too gay." As indicated at the bottom of Table 2, we calculated a measure that indicates the proportion of deans who agreed with at least one of the statements in the table. Over a third (35\%) of deans in our sample reported at least one negative climate issue for LGBTQ persons in their college.

\begin{tabular}{lr} 
Table 2: Proportion of Engineering Deans who Somewhat or Strongly Agree that the Following \\
Happen in their College (N=47) \\
\hline Coworkers make comments that seem to indicate a lack of awareness of LGBTQ issues & $21.74 \%$ \\
There is pressure for LGBTQ persons to stay closeted & $19.57 \%$ \\
LGBTQ persons must conceal their LGBTQ identity from colleagues & $14.89 \%$ \\
Employees are expected not to act "too gay" & $10.87 \%$ \\
The atmosphere for LGBTQ persons is oppressive & $4.35 \%$ \\
LGBTQ employees fear job loss because of sexual orientation or gender expression & $4.35 \%$ \\
LGBTQ persons are met with thinly veiled hostility & $2.17 \%$ \\
Proportion of Deans who agree that one or more of the above happens: & $\mathbf{3 4 . 7 8 \%}$ \\
\hline
\end{tabular}

Beyond deans' recognition of negative climate issues for LGBTQ persons, we were interested in the level of support deans expressed for a range of LGBTQ inclusion initiatives. Respondents were asked their level of support (1=very unsupportive to 5=very supportive) for the following initiatives: required addition of an LGBTQ-inclusive diversity statement on engineering course syllabi; college sponsorship of an oSTEM chapter (a student organization for LGBTQ engineering students); required gender-neutral restrooms in engineering buildings; Safe Zone training (training that provides information and skills for supporting LGBTQ faculty, students and staff) for interested engineering students; Safe Zone training for interested faculty; nondiscrimination statements for faculty job advertisements that included LGBTQ status; and hiring initiatives for an openly-LGBTQ faculty member.

The first bar in each grouping in Figure 1 presents the mean of deans' own support for these initiatives. We find that, on average, deans are somewhat or very supportive of six of the seven LGBTQ inclusion measures (i.e., the means for each are greater than 3.0). Deans are generally unsupportive of perhaps the most resource-intensive (or some may say radical) inclusion measure: hiring initiatives for openly-LGBTQ engineering faculty.

Our third research question examines deans' assessment of faculty and student support for this same set of initiatives. Deans may perceive a mismatch between their own supportiveness of these initiatives and the extent to which the faculty and students in their colleges and programs back such initiatives. 
Figure 1: Support for LGBTQ Inclusion Initiatives (1=Very Unsupportive to 5= Very Supportive; Bars Present Mean Values and $95 \%$ Confidence Intervals )

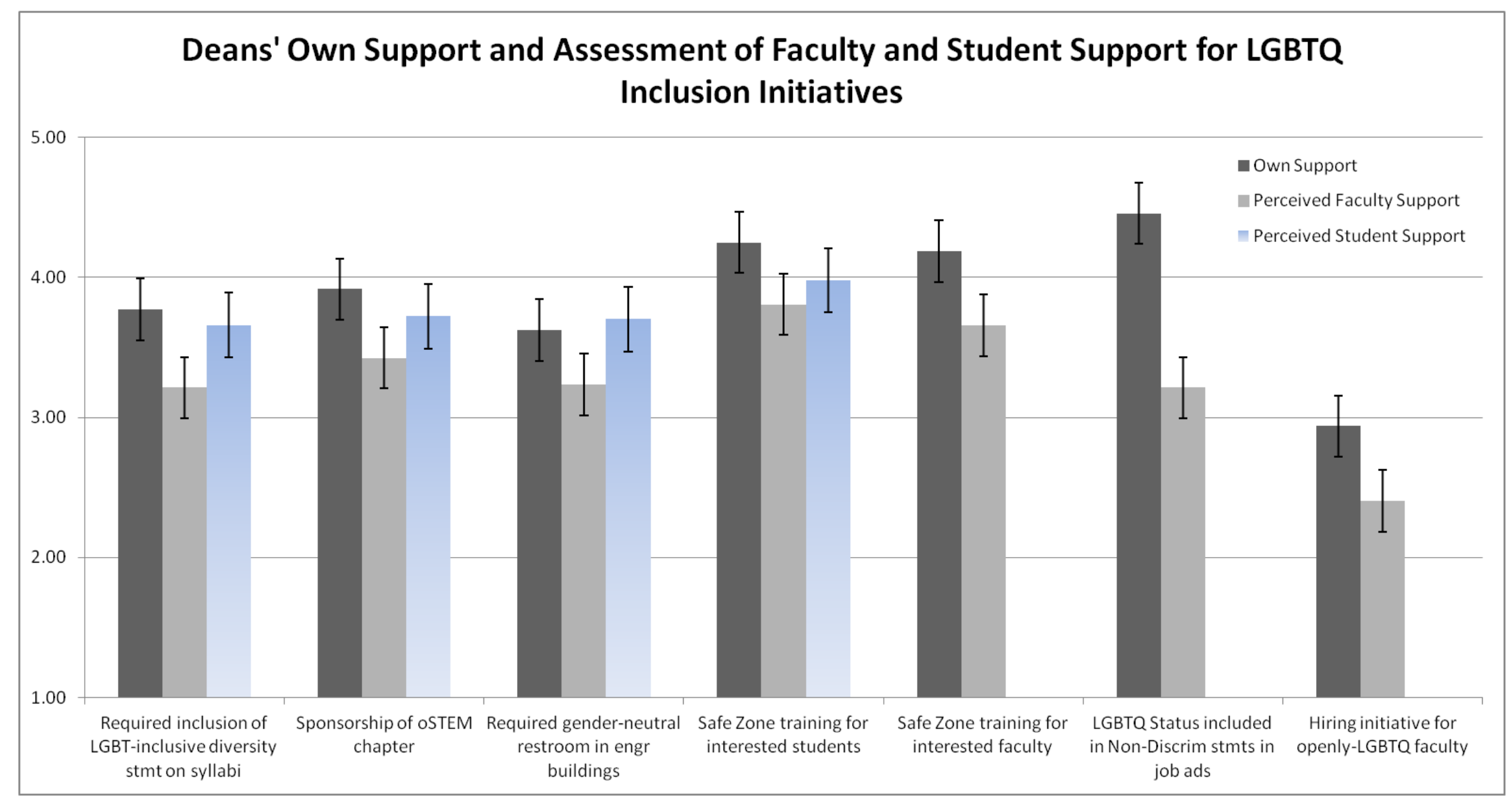


The second and third bar in each grouping in Figure 1 provides the means for deans' perceptions of the support among faculty for LGBTQ inclusion initiatives, and, where applicable, deans' perceptions of support among students. Compared to deans' personal support of LGBTQ inclusion initiatives, deans perceive faculty support for those same initiatives to be substantially weaker. Specifically, deans' perceptions of faculty support for LGBTQ-inclusive diversity statements on syllabi, sponsorship for an oSTEM chapter, Safe Zone trainings for interested faculty, LGBTQ-inclusive non-discrimination statements on job advertisements, and targeted hiring initiatives are significantly lower than their personal support for those initiatives. In contrast, the deans do not believe that engineering students are any less supportive of those initiatives than they are personally. We discuss the implications of these findings in the next section.

\section{Conclusions}

The purpose of this paper was to examine the recognition of LGBTQ inequality issues and the support for LGBTQ-inclusion initiatives among a population of institutional gatekeepers in higher education: engineering deans. Using a unique survey sample of over 40 deans, we examined the extent to which deans recognized issues of LGBTQ exclusion, marginalization, and heteronormativity within their engineering college. More than a third of deans reported that there was at least one aspect of LGBTQ marginalization within their college or program. Given the burgeoning literature documenting the challenges experienced by LGBTQ faculty and students in STEM [4,5,9-11], we expect that this is likely a substantial underestimation of the extent to which heterosexism and heteronormativity are present within U.S. engineering colleges. Nonetheless, in the absence of institutional-level initiatives and expectations for LGBTQ inclusion, deans' recognition of problems of LGBTQ inequality within their colleges is a necessary prerequisite for them to even consider initiating college-level changes that would advance LGBTQ inclusion.

We also were interested in deans' views on a variety of LGBTQ-inclusion initiatives such as adding LGBTQ status course syllabi diversity statements, to and Safe Zone trainings, to hiring initiatives for openly LGBTQ engineering faculty. We found that most deans expressed support for the majority of these initiatives. While expressing interest in such initiatives does not mean that deans are ready and willing to commit monetary and administrative resources to them, the strength of their support for these initiatives does suggest that engineering deans may be an underutilized resource for cultural and institutional changes that promote LGBTQ inclusion. Deans did not generally support hiring initiatives seeking openly-LGBTQ faculty, however.

Third, our analysis suggests an interesting differential between deans' own support for LGBTQ initiatives and their perceptions of faculty support for those initiatives. These results point to an important potential roadblock to the promotion of LGBTQ equality in engineering: Engineering deans may resist LGBTQ inclusion initiatives not because they are personally opposed to them but because they believe their faculty would be unsupportive of such initiatives.

These findings have a number of important implications. First, rather than serving as a roadblock to inclusion, the deans in our sample may be untapped allies in positive social change. Engineering students and faculty working "from the trenches" to improve the culture of engineering education for diverse groups of students and faculty may find more support than commonly assumed within engineering leadership. Second, demonstrations of faculty support 
for LGBTQ inclusion initiatives verbally and in writing may help give deans confidence that engineering faculty would be supportive of LGBTQ inclusion efforts. Finally, from an institutional perspective, finding ways to give engineering deans and other administrative leaders "cover" or institutional protection for the implementation of such initiatives may make deans more likely to consider risking pushback from faculty. Broadly, institution-wide implementation of LGBTQ inclusion programs, initiatives, and cultural expectations that include engineering programs may be a more effective means of widespread change. As such efforts have not manifest in most universities, such college-level efforts may be essential next steps.

Much is left to understand about the role of administrators in the promotion of LGBTQ inclusion in engineering. The next stage of our project will examine the experiences and attitudes of faculty and students in engineering. This research will illustrate not only the extent to which LGBTQ faculty and students feel marginalized within engineering education, but will also show whether faculty (LGBTQ and non-LGBTQ) are indeed less supportive of inclusion initiatives than their deans. Beyond this, research assessing the impact of college-level LGBTQ inclusion initiatives, such as the addition of an oSTEM chapter, LGBTQ inclusion statements on syllabi, or gender-neutral restrooms, on feelings of inclusiveness among LGBTQ individuals and the diminishment of LGBTQ bias among students and faculty would help us identify the most effective initiatives for cultural change.

\section{References}

1. National Academy of Sciences. (2007). Beyond Bias and Barriers: Fulfilling the Potential of Women in Academic Science and Engineering. In National Academy of Sciences. Committee on Maximizing the Potential of Women in Academic Science and Engineering, National Academy of Engineering, and Institute of Medicine. National Academies Press.

2. National Academy of Sciences. (2011). Expanding Underrepresented Minority Participation: America's Science and Technology Talent at the Crossroads: The National Academies Press.

3. National Science Foundation (2008) Women, Minorities and Persons with Disabilities in Science and Engineering. National Center for Science and Engineering Statistics. http://www.nsf.gov/statistics/wmpd/pdf/tab9-37.pdf

4. Cech, E. A. and T. J. Waidzunas. (2011). "Navigating the Heteronormativity of Engineering: The Experiences of Lesbian, Gay, and Bisexual Students." Engineering Studies. 3 (1): 1-24.

5. Riley, D. (2008). "LGBT-Friendly Workplaces in Engineering." Leadership Management Engineering. 8, special issue introduction, 19-23.

6. Ragins, B.R. and J.M. Cornwell. (2001). "Pink Triangles: Antecedents and Consequences of Perceived Workplace Discrimination against Gay and Lesbian Employees." Journal of Applied Psychology. 86(6):1244-61.

7. Ragins, B.R, R. Singh, and J.M. Cornwell. (2007). "Making the invisible visible: Fear and disclosure of sexual orientation at work." Journal of Applied Psychology. 92(4): 1103-1118.

8. Cech, E. A. and W. R. Rothwell. (2015). "Workplace Experiences among LGBT Employees" Working Paper. Rice University: Houston, TX.

9. Cech, E. A. (2015). "LGBT Professionals' Workplace Experiences in STEM-Related Federal Agencies. Proceedings of the 2015 American Society for Engineering Education (ASEE) National Conference. June 2015.

10. Bilimoria, D. and A. Stewart. (2009). “'Don't Ask, Don't Tell': The Academic Climate for Lesbian, Gay, Bisexual, and Transgender Faculty in Science and Engineering." NSWA Journal. 21 (2): 85-103. 
11. Patridge, E. V, R. Barthelemy, and S. R. Rankin. (2014). "Factors Impacting the Academic Climate for LGBTQ STEM Faculty." Journal of Women and Minorities in Science and Engineering. 20(1):75-98.

12. Cech, E. A. (2013). "The Veiling of Queerness: Depoliticization and the Experiences of LGBT Engineers." Proceedings of the 2013 American Society for Engineering Education (ASEE) National Conference. June 2013.

13. Jost, J.T., Banaji, M.R., \& Nosek, B.A. (2004) A Decade of System Justification Theory: Accumulated Evidence of Consious and Unconscious Bolstering of the Status Quo. Political Psychology, 25(6): 881-919.

14. Kluegel, J.R. \& Smith, E.R. (1986) Beliefs About Inequality: Americans' Views of What Is and What Ought to Be New York: Aldine de Gruyter.

15. Herek, G. M. (2007) "Confronting Sexual Stigma and Prejudice: Theory and Practice." Journal of Social Issues 63(4):905-25.

16. Cech, E. A. and H. M. Sherick. "Chapter 9: Depoliticization and the Structure of Engineering Education." International Perspectives on Engineering Education, ed. by Steen Hyldgaard Christensen et al. New York: Springer. Pp. 203-216. 